\title{
Entrelacs
}

Cinéma et audiovisuel

\section{Les multiples vies des séries télévisées : de la modification des logiques de programmation aux pratiques communautaires en ligne}

\section{Céline Ferjoux}

\section{OpenEdition}

\section{Journals}

Édition électronique

URL : http://journals.openedition.org/entrelacs/278

DOI : 10.4000/entrelacs.278

ISSN : 2261-5482

Éditeur

Éditions Téraèdre

\section{Édition imprimée}

Date de publication : 7 février 2008

ISSN : 1266-7188

Référence électronique

Céline Ferjoux, «Les multiples vies des séries télévisées : de la modification des logiques de

programmation aux pratiques communautaires en ligne », Entrelacs [En ligne], HS | 2008, mis en ligne le 01 août 2012, consulté le 10 décembre 2020. URL : http://journals.openedition.org/entrelacs/278 ; DOI : https://doi.org/10.4000/entrelacs.278

Ce document a été généré automatiquement le 10 décembre 2020.

Tous droits réservés 


\title{
Les multiples vies des séries télévisées: de la modification des logiques de programmation aux pratiques communautaires en ligne
}

\author{
Céline Ferjoux
}

1 Si l'on tente de considérer les différentes pratiques de réception et de consommation des séries télévisées, il est indispensable d'examiner un phénomène contemporain : la place occupée par les séries sur les sites internet des chaînes de télévision. L'observation de ce phénomène permet de mettre en exergue deux mouvements simultanés. L'un est lié à la position des chaînes et à leur objectif de captation des publics, et l'autre à des mécanismes d'usage propres aux médias interactifs.

\section{De l'acquisition aux logiques de programmation}

Du point de vue des chaînes, les séries constituent de nouveaux leviers d'audience. Ces contenus représentent un enjeu fondamental au niveau de l'acquisition des programmes. En France, les chaînes généralistes sont prêtes à payer le prix de la sécurité en se portant acquéreuses des séries qui ont déjà remporté un vif succès aux Etats-Unis. Les stratégies d'achat sont très fortement impactées par l'engouement pour ces formats étrangers. L'acquisition des séries américaines devient une activité à hauts risques ${ }^{1}$. Pour une chaîne privée (non payante) comme TF1 un indicateur influent est le coût de grille. Or pour l'année 2005 le groupe enregistre une hausse de 2,9\% du coût de la grille de l'antenne de TF $1^{2}$. Il est nécessaire de rappeler que les acteurs de l'audiovisuel se trouvent dans un contexte de positionnement stratégique très marqué. Les circonstances les amènent à prendre des risques pour conserver ou renforcer leurs positions dans le paysage audiovisuel. En se portant acquéreuses de séries populaires, les chaînes bénéficient d'un gain d'image qui leur permet de muscler l'identité de leurs marques auprès du public et 
des annonceurs. Eric Vérat (2007) explique très précisément les forces auxquelles elles sont soumises, « une chaîne qui fixe des tarifs publicitaires élevés pour une série attendue devra compenser l'échec d'audience s'il y a lieu ».

Pour compléter ce mouvement d'achat massif auprès des networks américains, et la logique de rentabilité dans laquelle elles se trouvent, les chaînes doivent offrir aux épisodes fraîchement acquis une place de choix dans leurs grilles et faire en sorte que les émissions rencontrent le public le plus large possible. En la matière l'année 2005 fut marquante puisqu'elle a vu apparaitre de nouvelles pratiques de programmation.

Comme le précisent Benoît Danard et Rémy Le Champion (2005), "programmer, c'est ordonnancer des émisssions dans une grille cohérente. Une fois édités, les programmes confèrent à la chaîne son caractère, son identité. ${ }^{4}$ " La programmation des grilles constitue le cœur de métier des chaînes de télévision. Les principaux enjeux professionnels et stratégiques concernent ces choix.

\section{Les cases perdues du grand écran}

3 Au cours de l'année 2005, un mouvement parallèle à la montée en puissance des séries américaines s'amorce, les chaînes accordent moins de place au cinéma sur les créneaux privilégiés de leurs grilles. Ce changement prend un caractère particulièrement risqué lorsqu'une des logiques de programmation est de ne pas bouleverser les habitudes du téléspectateur. Ainsi un rapport du CSA (repris par l'AFP) constate en mai 2005 : «L'offre de cinéma à la télévision évolue peu et les chaînes restreignent leur programmation de films ${ }^{5}$ ». L'article précise «alors que la fréquentation des salles de cinéma a atteint en 2004 son meilleur niveau depuis 20 ans, les quatre grandes chaînes nationales en clair ont réduit leur programmation cinématographique bien en deçà du nombre maximum de films autorisés par an ». Ce phénomène s'ajoute au choix de TF1 de programmer des séries américaines en prime time. La chaîne inaugure cette pratique avec la diffusion à $20 \mathrm{~h} 50$ le samedi soir de la première saison de Lost. Ces changements se multiplient jusqu'à atteindre un point culminant avec la déprogrammation du traditionnel «film du dimanche soir » annoncé avec les grilles de la rentrée 2006. Le 6 septembre 2006, Le Monde publie un article au titre explicite : «Le dimanche soir sur TF1, les films sont remplacés par les séries américaines». La modification des logiques de programmation est révélatrice des enjeux qui mobilisent le paysage audiovisuel français. Le succès est au rendez-vous. TF1 se félicite de ce choix en publiant un bilan élogieux des chiffres d'audience comme le montre un article de l'AFP du 21/08/20056, qui lui-même reprend un communiqué de presse adressé par la chaîne: "La série américaine à succès "Lost, les disparus » a battu ses records d'audience sur TF1 où ses deux derniers épisodes étaient diffusés samedi en première partie de soirée ». Les chiffres précisent que le dernier épisode de la saison a rassemblé 7,4 millions de téléspectateurs, soit une part d'audience de $38,1 \%$ du public âgé de 4 ans et plus. L'article ajoute « les derniers instants de cette série qui multiplie les énigmes ont attiré encore plus de téléspectateurs " puis reprend le communiqué où la chaîne précise qu'il y avait 8,2 millions de téléspectateurs «à $22 \mathrm{~h} 24$ pour connaître la fin de cette première saison ». TF1 annonce avoir réuni en moyenne $38,6 \%$ de part d'audience. En programmant la série Lost, TF1 consolide nettement sa position hégémonique sur les audiences de la première partie de soirée en battant notamment le jeu Fort Boyard diffusé à la même heure sur France 2. Avec près de $40 \%$ d'audience en prime time, TF1 consolide sa position de leader alors même que la 
concurrence de l'offre en matière de programmes explose (notamment avec le lancement de nouvelles chaînes gratuites sur le réseau numérique terrestre en mars 2005). La chaîne montre par le biais de la programmation des séries qu'elle sait prendre des risques et s'assure au passage de nouvelles recettes publicitaires. Il convient d'ajouter qu'auparavant les grilles estivales étaient délaissées et que le succès mondial des séries américaines a contribué à leur donner un nouveau souffle. Ce mouvement qui marque une présence plus discrète du cinéma parallèlement à un plus grand nombre de cases accordées à la fiction est souligné par le bilan annuel du $\mathrm{CSA}^{7}$. Le rapport précise que la fiction télévisuelle a gagné plus de 170 heures de diffusion annuelle et que sur les tranches de forte audience, 56 heures supplémentaires ont été programmées soit un total de plus de 300 heures de programmes entre $18 \mathrm{~h}$ et $23 \mathrm{~h}$. Le CSA précise : « cette évolution a principalement bénéficié à la fiction télévisuelle d'origine étrangère, notamment américaine.» La programmation des séries a bouleversé les logiques à l'œuvre auparavant. Cette modification des usages professionnels entraîne une adaptation au niveau des usages de réception puisque le public est amené à changer ses habitudes de consommation des programmes télévisés. Le succès d'audience de la série Lost diffusée sur TF1 à l'été 2005 apporte un exemple favorable à une vision évolutive des pratiques de programmation. Pour compléter cet aspect il faut noter que le format, le mode de narration et la richesse des univers proposés par les séries est propice aux performances d'audience.

\section{De la programmation aux dispositifs en ligne}

4 L'observation des logiques de programmation des séries télévisées peut être approfondie par une exploration des dispositifs web déployés par les chaines. Les choix de programmation opérés par les acteurs de l'audiovisuel trouvent un écho sur leur sites internet. Avec l'adoption massive d'équipements permettant l'accès aux contenus haut débit, les sites web des chaînes intègrent progressivement les stratégies des groupes audiovisuels. Les contenus proposés sur les pages d'accueil des sites reflètent les enjeux stratégiques et les lignes éditoriales construites par les chaînes. Les pages du site web sont le lieu de la réaffirmation du discours de la chaîne, de sa marque. A ce titre les émissions bénéficient d'une visibilité sur le site. Les choix en terme de programmation sont relayés sur le support internet et les contenus web participent à la familiarisation du public avec de nouveaux formats, de nouveaux univers fictionnels. Pour les networks américains, la stratégie consiste à accentuer, accompagner, encadrer les échanges qui peuvent se produire autour des séries populaires et de leur diffusion sur leurs réseaux. Il s'agit par exemple de proposer un dispositif web copieux prévoyant une programmation simultanée d'événements exclusifs réservés à leurs sites. Ainsi certaines séries font l'objet d'opérations événementielles sur le web et en 2006 apparaît également la mise à disposition en ligne de l'intégralité des épisodes, à peine la diffusion à l'antenne achevée. La logique de programmation événementielle des chaînes trouve une expression sur Internet. Cette continuité s'exprime par exemple à travers les campagnes publicitaires. Dans le cadre de la première diffusion de la série Lost sur TF1, la chaîne avait conclut deux contrats de parrainage du programme à l'antenne. D'après le bilan annuel établi par le $\mathrm{CSA}^{8}$, deux annonceurs étaient associés sur la totalité de la période de diffusion (du 25 juin au 20 août 2005) : il s'agissait de Eclipse Gum et AGF. Sur les pages du site de TF1 
consacrées à la série apparaissait un écran vidéo diffusant les spots publicitaires de la marque Eclipse Gum.

Écran 1

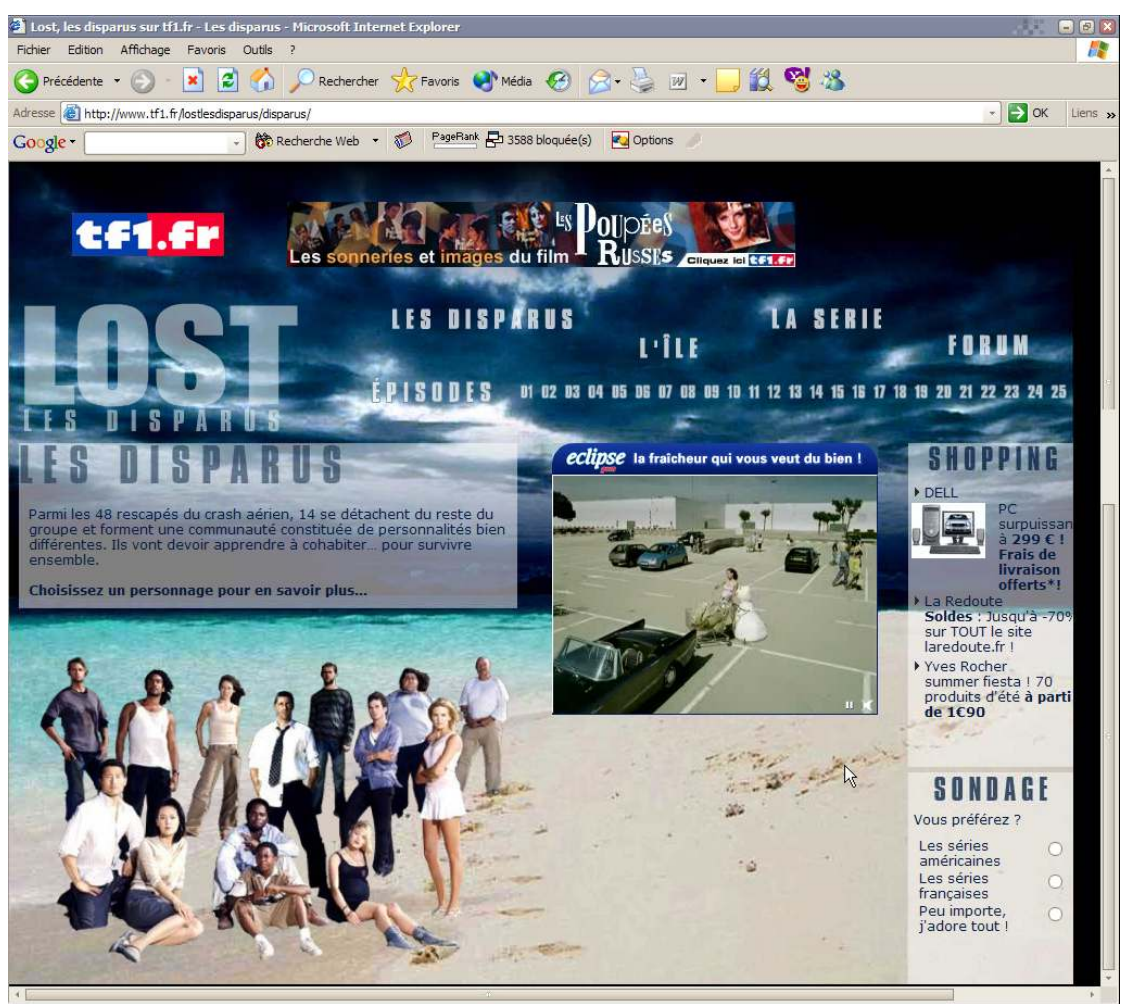

Mini-site Lost, page d'accueil, capture tf1.fr du 28/06/2005, 21 h00

Bien avant la diffusion du premier épisode de la première saison sur son antenne, TF1 met en place sur son site une campagne de teasing prenant pour objet la série. Un mois avant la diffusion à l'antenne, la page d'accueil du site de la chaîne propose une accroche accompagnée d'un visuel : "Lost. Les Disparus : bientôt sur TF1 ». Ces éléments occupent l'un des quatre emplacements de mises en avant au centre de la page d'accueil et donnent accès aux pages d'un site dédié à la période qui précède la diffusion. Ces pages proposent aux internautes de faire connaissance avec les différents protagonistes de la série, de découvrir la trame de l'histoire ainsi que le lieu où elle se déroule. Ce site structuré autour de trois entrées principales a pour vocation de familiariser le public-internaute avec l'univers de la série. Les contenus proposés sont assez sommaires, ils consistent essentiellement en des textes de présentation assez succincts. Deux galeries de photos sont accessibles. Il n'y a pas de forum, les internautes ont cependant la possibilité de s'exprimer dans la fenêtre d'un sondage qui les interpelle : «Vous préférez ? / Les séries américaines / Les séries françaises / Peu importe, j’adore tout!». Le 6 juin 2005, 2055 internautes ont voté. A $81 \%$ ils ont choisi les séries américaines. 
Écran 2

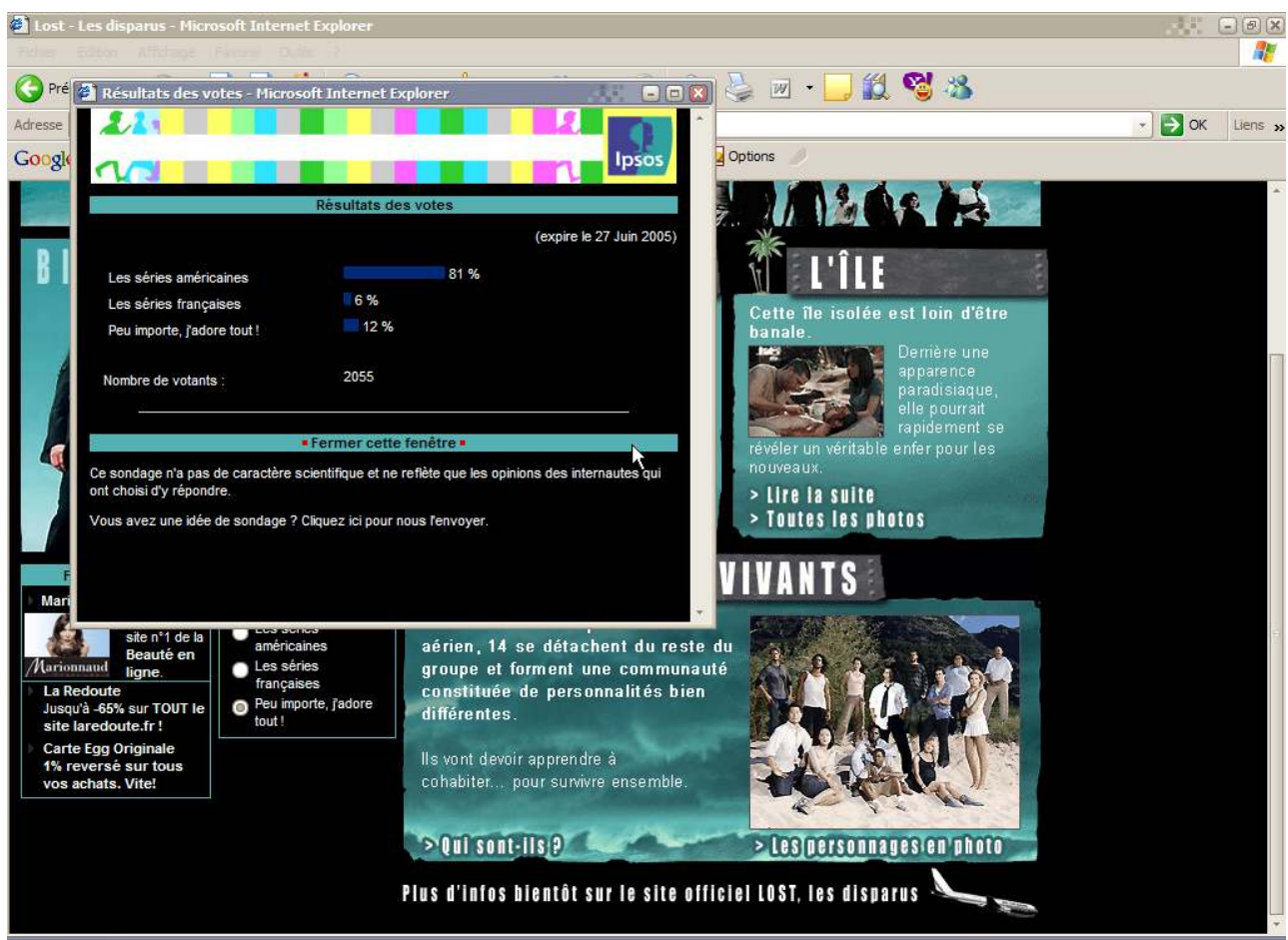

Mini-site Lost, fenêtre sondage, capture tf1.fr du 06/06/2005, 19h00

6 TF1 propose un espace consacré à la série bien avant la diffusion et elle l'intègre à sa page d'accueil. La chaîne offre ainsi des éléments de familiarisation à son public-internaute, dont elle aiguise la curiosité tout en associant sa marque à la renommée et à l'univers de la série. A ce titre le discours employé pour cette promotion avant la diffusion est sans équivoque, les visuels présents sur le site associent de manière systématique le logo de la chaîne à celui de la série. Comme le montre cette accroche présente à la Une de la page d'accueil : « Lost / Les Disparus / Ils arrivent sur [logo TF1] ». Cette logique s'intensifie et peu de temps avant la diffusion, la page d'accueil est entièrement mise aux couleurs de Lost et intègre un décompte des jours restants avant la première émission. 


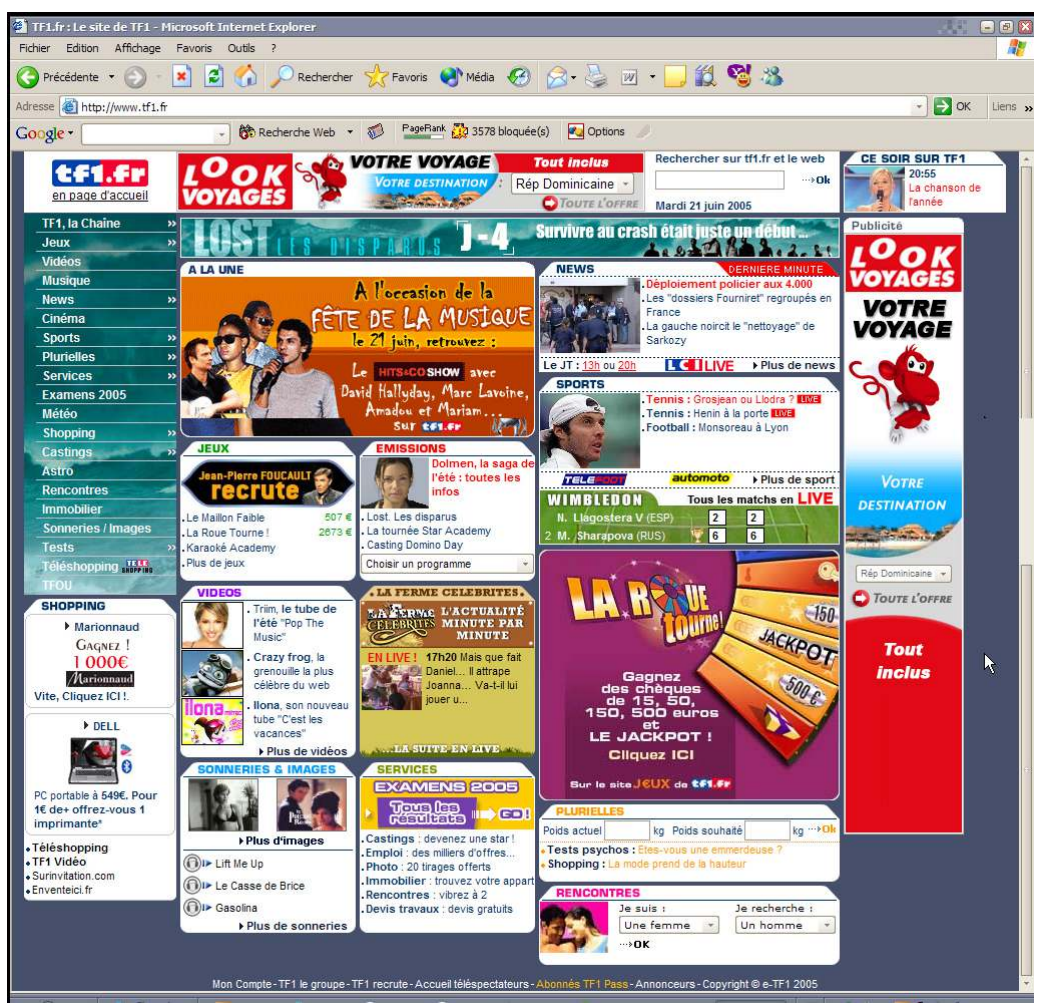

Page d'accueil, capture tf1.fr du 21/06/2005, 18h30

7 La maquette de la page d'accueil a été modifiée pour l'occasion, un bandeau a été ajouté entre le header publicitaire et la Une. Il porte la mention : «Lost / Les disparus / J-4 / Survivre au crash était juste un début... ». Cet élément renvoie aux pages du pré-site. La chaîne conçoit donc un dispositif varié et massif de promotion de la série dont elle s'est portée acquéreuse. Le web représente ainsi un espace privilégié d'acclimatation pour les téléspectateurs-internautes curieux de découvrir une série avant même qu'elle ne soit diffusée à l'antenne. Il serait délicat d'aller plus loin dans la description du dispositif web mis en place par TF1 au sein de ce court article. Néanmoins il est essentiel de souligner son ampleur. Pour les chaînes de télévision, la programmation des séries représente un enjeu majeur de fidélisation de l'audience. Elles exploitent la temporalité des séries, suivent le rythme des « saisons » et utilisent à cette fin le support web pour assurer une continuité entre les phases de diffusion saccadées et soumises au rythme de production de leurs homologues d'outre-Atlantique. Parallèlement, le format et les codes véhiculés par les séries télévisées semblent propices à fertiliser les pratiques communautaires en ligne.

\section{Les pratiques communautaires en ligne : se perdre pour mieux se reconnaître}

8 L'exemple de dispositif de teasing observé montre que TF1 investit précosément le support internet. De cette manière, la chaîne maîtrise une partie du discours relatif au programme. La nature des contenus qui circulent sur le web est vouée à s'étoffer progressivement au cours du cycle de vie de la série. Avant la diffusion, les informations 
disponibles sont relativement sommaires et leur volume est limité. Elles concernent principalement l'identité des personnages, la trame de l'histoire et plantent le cadre global de la narration. Les producteurs veillent cependant à fournir des éléments capables d'irriguer l'imagination des amateurs de séries télévisées. Avec le succès d'audience du programme les internautes s'emparent du sujet. Diverses composantes du format sériel sont propices à la production d'activités en réseau. L'univers d'une série américaine comme Lost recèle des ingrédients de nature à amener les spectateurs sur le terrain de la prospective. La construction des épisodes maintient un niveau de suspense élevé et soulève de nombreuses énigmes. La narration a recours à l'ellipse et entretient le mystère d'une émission à l'autre. Ces interstices laissent libre court aux prospectives des téléspectateurs-internautes. En parallèle de la diffusion de la série, les scénaristes et producteurs organisent un jeu qui consiste à rassembler les pièces d'un puzzle. A ce titre, le projet The Lost Experience, founit une illustration de la volonté des networks d'alimenter les pratiques en ligne. Au printemps 2006, $\mathrm{ABC}$ lance une quête mondiale à partir du site www.thelostexperience.com, elle invite les internautes à résoudre les mystères de l'île des naufragés ${ }^{9}$. Le scénario des saisons successives de Lost sera en parallèle l'objet de quêtes interactives en ligne. Les créateurs associent à la diffusion télévisée des épisodes une distribution d'indices sur la constellation de sites construits autour de The Lost Experience.

Écran 4

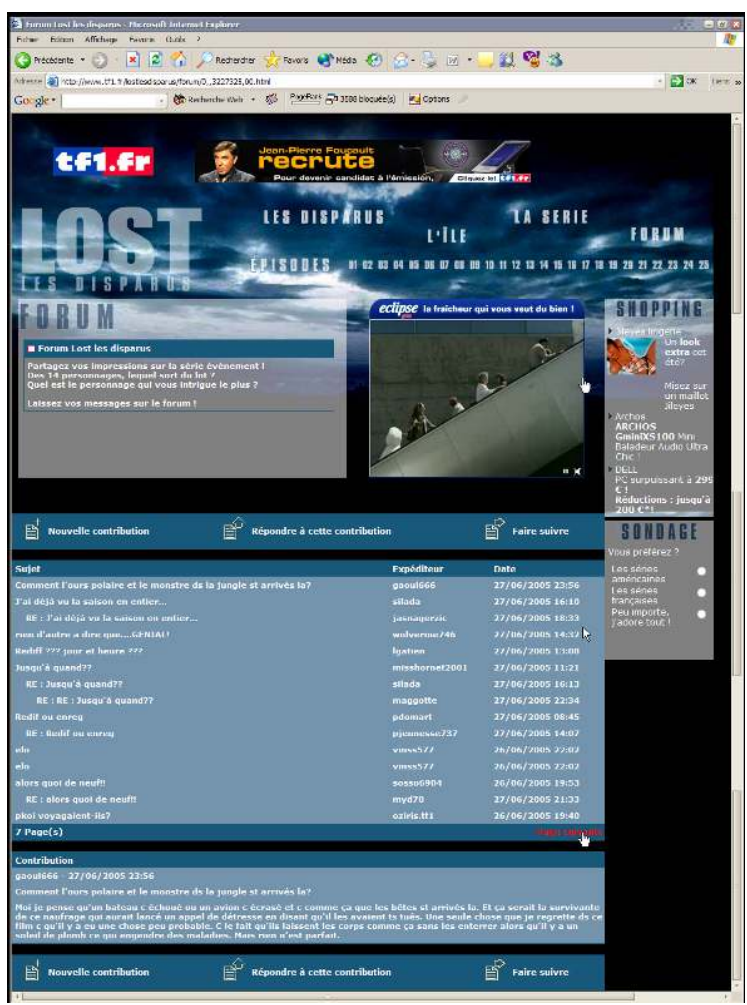

Site The Hanso Foundation, page d'accueil, capture www.thehansofoundation.org du 06/08/2007, $20 \mathrm{h00}$ 


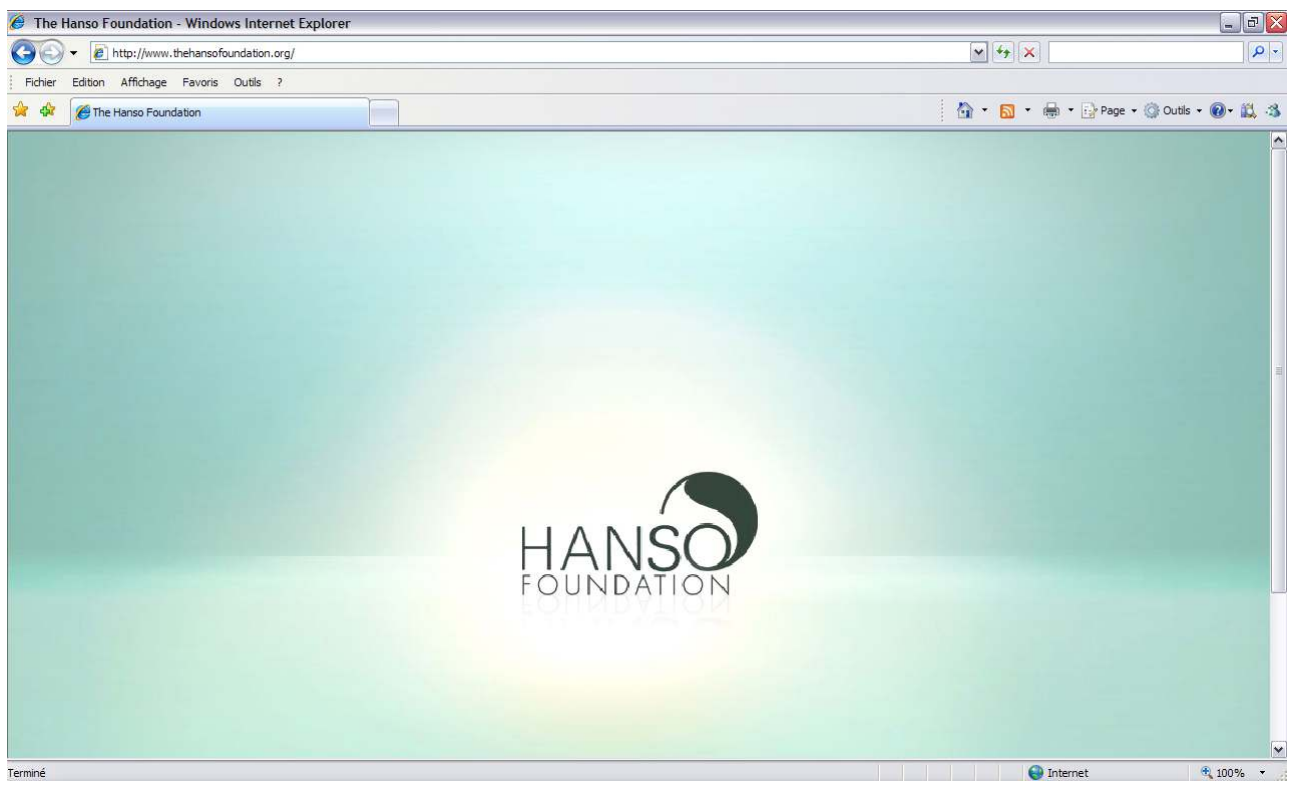

Site The Lost Experience, page d'accueil, capture www.thelostexperience.com du 06/08/2007, 20h00

Un autre ingrédient propre à fertiliser les pratiques communautaires en ligne est la psychologie des personnages. Les concepteurs de séries télévisées travaillent à la création de personnages de fiction à la psychologie plus subtile et dense que ne le sont les figures du cinéma. L'espace temporel ouvert des fictions sérialisées leur permet d'installer et de développer des personnalités plus riches et moins stéréotypées que celles qui prennent corps le temps d'un film au cinéma. Les scénaristes créent des personnages complexes qui favorisent les mécanismes d'identification et de réappropriation auprès des internautes notamment. De telles logiques aboutissent à de nouvelles pratiques dont font partie les «fansfictions ». Cet aspect du formatage des séries est décrit par Martial Martin (2007) ${ }^{10}$ : «c'est là ce qui fascine ces internautes et ce qu'ils tentent de reconduire à travers leurs propres fictions: interpeller le plus grand nombre par le biais de personnages très largement connus et d'intrigues souvent convenues [...] les continuations des séries télévisées ne visent pas la distinction, mais semblent exalter le plaisir du partage le plus large ». Du point de vue des internautes-téléspectateurs, la consultation des contenus proposés sur les sites web représentent un moyen privilégié de poursuivre l'expérience de réception des séries. La consommation de ces nouveaux formats audiovisuels se décline sur Internet et déborde le temps de l'émission des programmes tout en prenant appui sur cette diffusion. L'observation d'un échantillon des sujets abordés sur le forum consacré à Lost sur le site de TF1 en atteste. Sur la première page du forum qui propose les quinze derniers sujets de discussion, la moitié font clairement référence à la diffusion. Certains, par exemple, questionnent les membres du forum sur les conditions de rediffusion des épisodes ou bien sur la possibilité d'obtenir une copie d'un enregistrement. La diffusion de la série à l'antenne et les réactions postées sur le forum sont donc étroitement liées. 
Écran 6

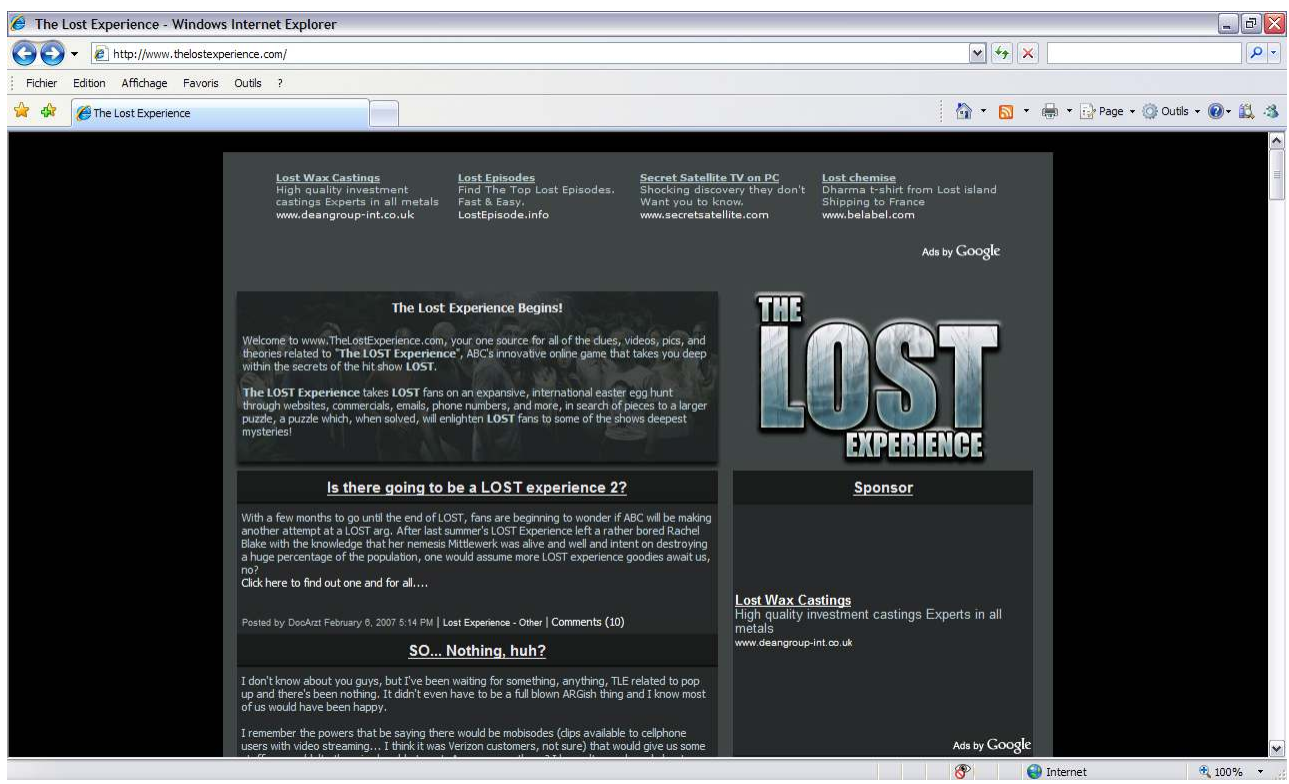

Mini-site Lost, page forum (1), capture tf1.fr du 28/06/2005, 21 h00

10 Le site de la chaîne permet aux téléspectateurs-internautes de combler la frustration créée par le dispositif télévisuel et la construction narrative de la série. Il permet aux internautes de prolonger l'expérience d'immersion dans la fiction tout en leur offrant l'illusion d'une participation au processus créatif, ou au moins, une consommation sur le mode actif de la série et de son univers. Une étude plus approfondie de ces phénomènes pourrait être entreprise dans un cadre plus large que celui d'un court article qui tente d'esquisser la direction de recherches entreprises par ailleurs. Les récents travaux autour de la constitutions des communautés virtuelles et menés par Serge Proulx (2006) ${ }^{11}$ peuvent servir d'appui à la description des mécanismes d'usage liés à la consommation des séries télévisées sur les sites internet des chaînes de télévision. Ces programmes construisent des univers fictionnels contemporains riches d'éléments propres à fertiliser les pratiques communautaires, virtuelles et volatiles, qui animent le réseau Internet. Notamment en raison de leur capacité à déclencher les processus d'identification, conditions nécessaires à la création de groupes d'affinités. Comme l'explique Serge Proulx $(2006)^{12}$, «entre les membres de la communauté, il y a nécessairement un partage de croyances idéologiques et d'habitudes culturelles, de valeurs communes, d'un sens de la solidarité et de l'identification à une même constellation identitaire d'appartenance (construction d'un sentiment d'identité à un nous commun). » Ces communautés sont néanmoins volatiles car les membres, même s'ils sont soumis à des procédures d'inscription ou d'abonnement, partagent des informations et ensuite ont la possibilité de se disperser pour peut-être ne jamais poursuivre les échanges.

11 En tant que contenus privilégiés ayant un fort retentissement sur le réseau, les séries dépassent l'enjeu pour les acteurs de l'audiovisuel de maintenir leur marque et renforcer leur identité de puissances médiatiques. Finalement, l'engouement fantastique pour ces programmes pourrait en partie s'expliquer par leur nature propre à fertiliser les pratiques communautaires des médias interactifs et numériques. 


\section{NOTES}

1. AFP, Rubrique Médias. 04/10/2006, 07h54. S'emparer de la série US qui fera date? Une question de flair et de rapidité

2. Groupe TF1. Rapport annuel 2005 : www.tf1finance.fr/documents/publications/185.pdf

3. Eric Vérat (2007), « Etats-Unis : le règne des saisons et la galaxie des auteurs ». Médiamorphoses Hors Série, Les raisons d'aimer les séries télé. INA, Armand Colin. janv.2007, pp.18-23.

4. Benoît Danard, Rémy Le Champion (2005) Les programmes audiovisuels. coll. Repères, ed. La Découverte, Paris, juin 2005, p.52

5. AFP, Rubrique Médias. 04/05/2005, 15h27. «De moins en moins de films sur les chaînes de télévision françaises"

6. AFP, Rubrique Médias. 21/08/2005, 12h23. «TF1 : Lost bat ses records d'audience avec ses deux derniers épisodes "

7. CSA, Bilan de la société privée TF1. Année 2005: www.csa.fr/upload/publication/ bilantf12005.pdf

8. id.

9. AFP, Rubrique Médias. 26/04/2006, 06h32. «Une quête sur l'internet pour les fans de Lost - Les disparus ».

10. Martial Martin (2007), «Les fansfictions sur Internet ». Médiamorphoses Hors Série, Les raisons d'aimer les séries télé. INA, Armand Colin. janv.2007. pp. 186-189.

11. Serge Proulx (2006) «Les communautés virtuelles: ce qui fait le lien». In: Communautés virtuelles - Penser et agir en réseau. coll. Laboratoire de communautique appliquée, Presses universitaires de Laval. 2006. 361p, pp. 13-25

12. id. p. 19

\section{RÉSUMÉS}

Pour considérer les pratiques de réception et de consommation des séries télévisées, il est indispensable d'examiner un phénomène contemporain : la place occupée par les séries sur les sites internet des chaînes de télévision. L'observation de ce phénomène permet de mettre en exergue deux mouvements simultanés. L'un est lié à la position des chaînes et à leur objectif de captation des publics, et l'autre à des mécanismes d'usage propres aux médias interactifs. Du point de vue des chaînes, les séries représentent de nouveaux leviers d'audience et amènent les acteurs de l'audiovisuel à modifier leurs pratiques en matière de programmation. Au cours de l'année 2005, TF1 prend le risque de boulever ses grilles estivales. Le public est au rendez-vous. Les logiques de programmation traditionnelles évoluent et trouvent un écho sur le web. Les chaînes utilisent leur sites pour déployer l'univers des séries. Le format de ces programmes est propice pour voir éclore de nouveaux usages. Du point de vue des téléspectateurs-internautes, les contenus proposés sur le web leur permettent de poursuivre l'expérience de réception des séries. La consommation du programme déborde le temps de la diffusion télévisée. Sur quels 
mécanismes d'usages s'appuie cette forme de réception? Quels rôles y jouent les pratiques communautaires (virtuelles et volatiles) et dans quelles mesures les médias interactifs participent de ces pratiques? Voici quelques-unes des pistes qui seront explorées dans cet article. Finalement, l'engouement pour les séries ne pourrait-il pas s'expliquer par leur nature propre à fertiliser les pratiques communautaires des médias interactifs ?

\section{AUTEUR}

\section{CÉLINE FERJOUX}

Doctorante CARISM, Université Paris II (IFP) 